\title{
A Novel Method for Obtaining a Crack Tip Appropriate Multiaxiality Constant for Damage Mechanics
}

\author{
Christopher J. Hyde ${ }^{1, *}$, Wei Sun ${ }^{1}$ and Thomas H. Hyde ${ }^{1}$ \\ 1. Department of Mechanical, Materials and Manufacturing Engineering, The University of Nottingham, Nottingham, NG7 2RD, UK
}

Received June 5, 2009 / Accepted September 9, 2009 / Published January 8, 2010.

\begin{abstract}
Many engineering components, such as power-plant steam pipes, aero-engine turbine discs, etc, operate under severe loading/temperature conditions. As a result, cracks can initiate and subsequently propagate over time due to creep. The Liu and Murakami model has proven to be a useful tool for the prediction of creep crack growth under such conditions. Previously, experimental conditions used in obtaining the constant of multiaxiality, $\alpha$, have not reflected the multiaxial severity of the stress-state ahead of a crack tip. Therefore, the present study presents a novel method for interpolating crack growth data to obtain $\alpha$.
\end{abstract}

Key words: Damage, multiaxiality, creep, crack growth.

\section{Introduction}

Components in power plant, chemical plant, manufacturing processes, aeroengines, etc may operate at temperatures which are high enough for creep to occur [1]. Such components may contain cracks or must be assumed to contain cracks as part of design life or remaining life analyses which are required [2]. In order to perform these analyses a number of approaches have been used, based on, for example, a fracture mechanics approach using $\dot{a}-C^{*}$ relationships [3], or a damage mechanics approach [4, $5,6]$. This paper is related to the use of the damage mechanics approach, methods used to obtain the material constants and comparisons of the predictions to experimental data. Most of the constants are obtained by fitting to uniaxial creep data, see Fig.1; this is a well-established method [7]. However, in this paper, the determination of the multiaxial stress state parameter, $\alpha$ [8], is based on the test results obtained using compact tension (CT) specimens, Fig.2; this approach is novel and results in properties which are particularly suited for predicting creep crack growth in components, where the crack growth is defined by a damage parameter, $\omega$. When this damage parameter reaches a critical value ( 0.99 chosen for the presented work) the material is regarded as 'completely damaged' and hence a void or crack growth is assumed to be present. A previously used technique for obtaining the multiaxial stress state parameter, based on the notch strengthening which usually occurs in Bridgman notch [9] creep rupture tests, relative to corresponding uniaxial tests, does not closely represent the stress states and constraint which occur at crack tips. The validity of the method proposed in this paper is established by comparing Finite Element $(F E)$ predictions of creep crack growth in thumbnail cracked specimens, see Fig.3, with experimental data [7] using the material constants obtained from uniaxial creep and $C T$ creep test results. All of the results presented within this paper are for 316 stainless steel at $600^{\circ} \mathrm{C}$. 


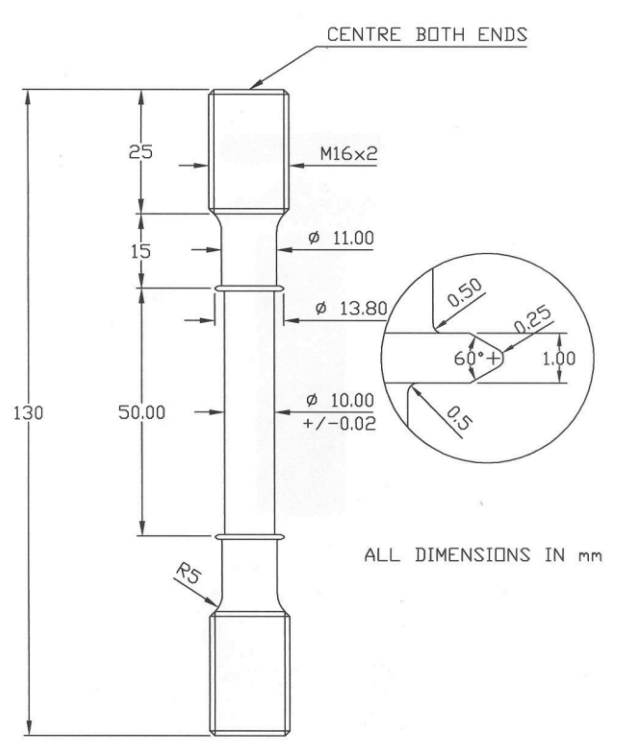

Figure 1. Uniaxial creep specimen geometry.
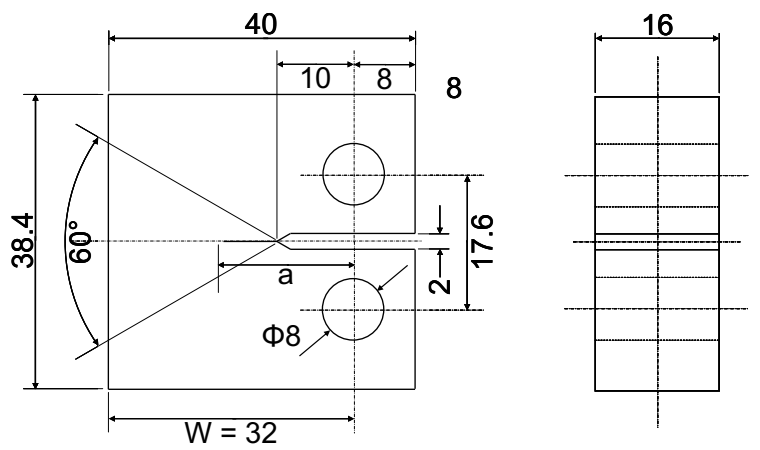

$\mathrm{a} / \mathrm{W}=0.5$

Figure 2. $C T$ creep crack growth specimen geometry (dimensions in $\mathrm{mm}$ ). (a)

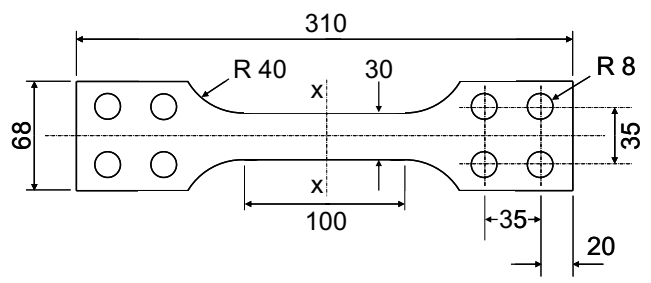

(b)

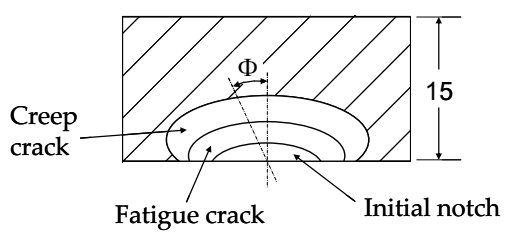

Figure 3. Thumbnail creep crack growth specimen geometry (a) side-on view, and (b) an enlarged section view through the $X-X$ plane (dimensions in $\mathrm{mm}$ ).

\section{Liu \& Murakami creep damage model}

\subsection{Definition of the material model}

The multiaxial form of the Liu and Murakami creep damage law is as follows:

$$
\dot{\varepsilon}_{i j}^{c}=\frac{3}{2} C \sigma_{e q}^{n_{2}} \frac{S_{i j}}{\sigma_{e q}} \exp \left(\frac{2\left(n_{2}+1\right)}{\pi \sqrt{1+3 / n_{2}}} \cdot\left(\frac{\sigma_{1}}{\sigma_{e q}}\right) \cdot \omega^{3 / 2}\right)
$$

where $C$ and $n_{2}$ are material constants. $\varepsilon_{i j}^{c}$ and $\sigma_{e q}$ are the creep strain tensor and the von-Mises equivalent stress, respectively, and $\sigma_{l}$ is the maximum principle stress. $S_{i j}$ is the deviatoric stress tensor, i.e.:

$$
S_{i j}=\sigma_{i j}-\frac{1}{3} \delta_{i j} \sigma_{k k}
$$

in which $\delta_{i j}$ is the Kronecker delta and is defined as:

$$
\delta_{i j}= \begin{cases}1 & i=j \\ 0 & i \neq j\end{cases}
$$

where $\sigma_{h}$ is the hydrostatic stress, defined as:

$$
\sigma_{h}=\frac{\sigma_{11}+\sigma_{22}+\sigma_{33}}{3},
$$

$\omega$ is the damage variable, and its rate of change with time is given as: 


$$
\dot{\omega}=D \frac{\left(1-e^{-q_{2}}\right)}{q_{2}} \sigma_{r}{ }^{p} e^{q_{2} \omega}
$$

When this value reaches a critical value (0.99 within the present work), crack growth is assumed to have occurred into the regions where this has happened. $D$, $q_{2}$ and $p$ are material constants. $\sigma_{r}$ is the rupture stress defined as:

$$
\sigma_{r}=\alpha \sigma_{1}+(1-\alpha) \sigma_{e q}
$$

where $\alpha$ is a material constant which describes the effect of multi-axial stress states.

Under the uniaxial condition:

$$
\sigma_{1}=\sigma_{e q}=\sigma
$$

and $\sigma_{11}$ can be substituted for $\sigma_{l}$.

Therefore, substituting equation (6) into equation (5) gives:

$$
\sigma_{r}=\sigma_{e q}
$$

and therefore, from equation (6):

$$
\sigma_{1}=\sigma_{e q}=\sigma_{r}=\sigma
$$

Hence, under the uniaxial condition, it is not possible to determine the material constant, $\alpha$. Also, for the uniaxial condition (the 11-direction), equation (2) can be simplified to:

$$
S_{11}=\sigma-\frac{1}{3} \sigma=\frac{2}{3} \sigma
$$

as $\sigma_{22}$ and $\sigma_{11}$ are both equal to zero and $\sigma_{11}=\sigma_{1}=\sigma_{e q}=\sigma$ under the uniaxial condition. Therefore, substituting equations (8) and (6) into equation (1) gives:

$$
\dot{\varepsilon}^{c}=C \sigma^{n_{2}} \exp \left(\frac{2\left(n_{2}+1\right)}{\pi \sqrt{1+3 / n_{2}}} \cdot \omega^{3 / 2}\right)
$$

Also, for the uniaxial condition, equation (7) can be substituted into equation (4) to give:

$$
\dot{\omega}=D \frac{\left(1-e^{-q_{2}}\right)}{q_{2}} \sigma^{p} e^{q_{2} \omega}
$$

Equations (9) and (10) are the uniaxial form of the Liu and Murakami damage model.

\subsection{Determination of the material constants}

From equations (1), (4) and (5), it can be seen that the constants which are required to be obtained are $C, n_{2}, D, q_{2}, p$ and $\alpha$. Methodologies for obtaining these constants are described as follows:

1.2.1. Uniaxial material constants $(\mathrm{C}, \mathrm{n} 2, \mathrm{D}, \mathrm{q} 2$ and $\mathrm{p})$

\section{$C$ and $n_{2}$}

During the initial stages of the creep of a material, $\omega \approx 0$ and hence $\exp \left(\frac{2\left(n_{2}+1\right)}{\pi \sqrt{1+3 / n_{2}}} \cdot \omega^{3 / 2}\right) \approx \exp (0)=1 \quad \cdot \quad$ Therefore equation (9) can be simplified to the following:

$$
\dot{\varepsilon}^{c}=C \sigma^{n_{2}}
$$

Taking $\log$ s of both sides of equation (11) gives,

$$
\log \left(\dot{\varepsilon}^{c}\right)=n_{2} \log (\sigma)+\log (C)
$$

Therefore, using experimental uniaxial creep data to plot $\log \left(\dot{\varepsilon}^{c}\right)$ vs. $\log (\sigma)$ and fitting a straight line of best fit through this data allows the identification of $n_{2}$ from the gradient and $C$ from the intercept. An example of this plot is shown in Fig.4, for 316 stainless steel, at $600^{\circ} \mathrm{C}$. The $C$ and $n_{2}$ values determined for the 316 stainless steel, at $600^{\circ} \mathrm{C}$, are included in Table 2. 


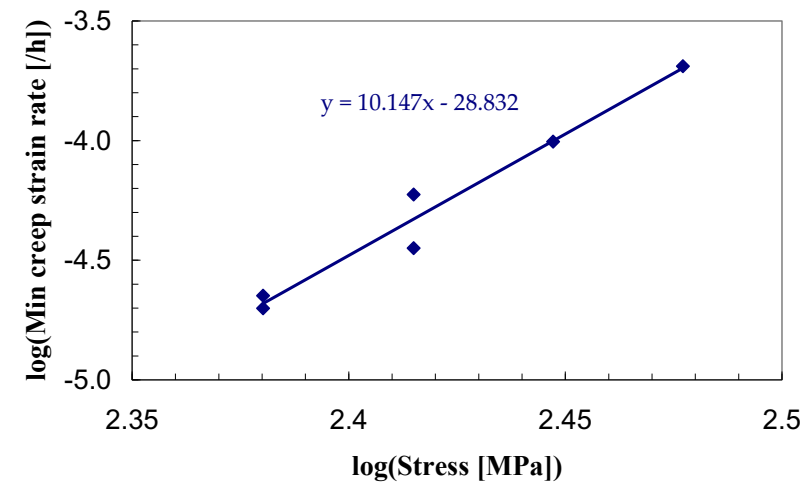

Figure 4. Linear fit to minimum creep strain rate vs. stress on a $\log -\log$ scale.

\section{$D$ and $p$}

Equation (10) can be written as follows:

$$
\frac{d \omega}{d t}=D \frac{\left(1-e^{-q_{2}}\right)}{q_{2}} \sigma^{p} e^{q_{2} \omega}
$$

Separating the variables for integration gives:

$$
\frac{d \omega}{e^{q_{2} \omega}}=D \frac{\left(1-e^{-q_{2}}\right)}{q_{2}} \sigma^{p} d t
$$

Since the right hand side of equation (12) is made up entirely of constants, equation (12) can be rewritten as:

$$
\frac{d \omega}{e^{q_{2} \omega}}=Q d t
$$

where $Q=D \frac{\left(1-e^{-q_{2}}\right)}{q_{2}} \sigma^{p}=$ constant

Integrating equation (13) between the limits of 0 and 1 , for $\omega$, and 0 and $t_{f}$, for $t$ gives:

$$
\int_{0}^{1} e^{-q_{2} \omega} d \omega=Q \int_{0}^{t_{f}} d t
$$

Equation (15) can be further simplified using the substitution:

$$
-q_{2} \omega=\Omega
$$

and therefore $\quad-q_{2} d \omega=d \Omega$

Therefore, equation (15) becomes:

$$
\int_{0}^{-q_{2}} \frac{e^{\Omega}}{-q_{2}} d \Omega=Q \int_{0}^{t_{f}} d t
$$

Note that the damage integration limits have also changed as a result of the substitution shown by equations (16) and (17). Equation (18) can be solved to give:

$$
\frac{1-e^{-q_{2}}}{q_{2}}=Q t_{f}
$$

Substituting equation (14) into equation (19) gives the following:

$$
t_{f}=\frac{\sigma^{-p}}{D}
$$

Taking logs of both sides of equation (20) gives:

$$
\log \left(t_{f}\right)=-p \log (\sigma)+\log \left(\frac{1}{D}\right)
$$

Therefore, plotting $\log \left(t_{f}\right)$ vs. $\log (\sigma)$ using data obtained from uniaxial creep tests, allows the identification of both $p$, from the gradient of the straight line of best fit and $D$, from the y-intercept. Fig. 5 shows an example of this plot for uniaxial, 316 stainless steel data at $600^{\circ} \mathrm{C}$. The $D$ and $p$ values obtained for the 316 stainless steel, at $600^{\circ} \mathrm{C}$, are included in Table 2.

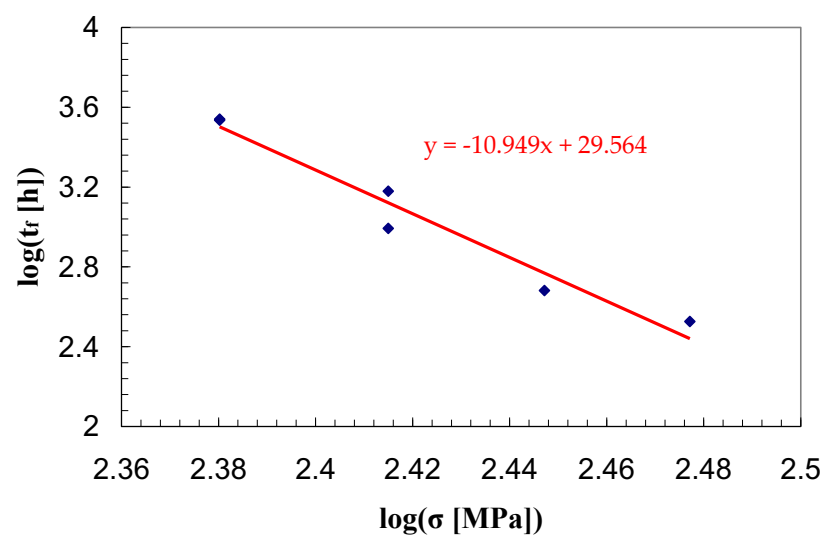

Figure 5. Linear fit to $\log (\mathrm{tf})$ vs. $\log (\sigma)$. 
$q_{2}$

At this stage, all of the constants required in the uniaxial version of the Liu and Murakami model are known, except the $q_{2}$ value. A curve fitting process is used on the $\varepsilon^{c}$ vs. time data in order to determine the value of $q_{2}$ which is the optimum fit at all stress levels.

In order to plot $\varepsilon^{c}$ vs. time using the model, $\varepsilon^{c}$ must first be found as a function of $t$. As equation (9) shows, $\dot{\varepsilon}^{c}$ is a function of $\omega$ as well as $t$. $\omega$ is also a function of $t$, as shown by equation (10). Therefore, this expression for $\omega$ as a function of $t$ must first be found, which can then be substituted into equation (9) to give an expression for $\varepsilon^{c}$ as a function of $t$. Integrating equation (13), between the limits of 0 and $\omega$, for $\omega$, and 0 and $t$, for $t$, leads to an expression for $\omega$ as a function of $t$, i.e.,

$$
\int_{0}^{\omega} \frac{d \omega}{e^{q_{2} \omega}}=Q \int_{0}^{t} d t
$$

Again, using the substitution shown by equations (16) and (17) gives:

$$
\int_{0}^{-q_{2} \omega} \frac{e^{\Omega}}{-q_{2}} d \Omega=Q \int_{0}^{t} d t
$$

Equation (23) can be solved and re-arranged for $\omega$ to give:

$$
\omega=-\frac{\ln \left(1-Q q_{2} t\right)}{q_{2}}
$$

Substituting equation (14) into equation (24) gives:

$$
\omega=-\frac{\ln \left(1-D\left(1-e^{-q_{2}}\right) \sigma^{p} t\right)}{q_{2}}
$$

This is the expression for $\omega$ (as a function of $t$ ) which is needed in order to obtain an expression for $\varepsilon^{c}$ as a function of $t$. Equation (25) is substituted into equation (9) to give:

$$
\begin{gathered}
\dot{\varepsilon}^{c}=C \sigma^{n_{2}} \exp \left(\frac{2\left(n_{2}+1\right)}{\pi \sqrt{1+3 / n_{2}}} \ldots\right. \\
\ldots \times\left(-\frac{\ln \left(1-D\left(1-e^{-q_{2}}\right) \sigma^{p} t\right)}{q_{2}}\right)^{3 / 2}
\end{gathered}
$$

Equation (26) cannot be readily solved to produce a closed form solution for $\varepsilon^{c}$ as a function of time. However, a time marching procedure can be used to obtain the variation of $\varepsilon^{c}$ with time. This time marching procedure is carried out by calculating $\dot{\varepsilon}^{c}$ for many small time steps, up to the failure time, and multiplying each of these values by the small time interval in order to give the creep strain increment for that time interval, as shown by the following equation:

$$
\Delta \varepsilon_{i}^{c}=\dot{\varepsilon}_{i}^{c} \times \Delta t
$$

where $i$ denotes the current time step. These creep strain increments are then accumulated in order to give the total value of creep strain at the end of each time step, i.e.

$$
\varepsilon_{i}^{c}=\varepsilon_{i-1}^{c}+\Delta \varepsilon_{i}^{c}
$$

Stress, $\sigma$, is assumed to be constant for every time increment. Curves of $\varepsilon^{c}$ vs. $t$ can then be plotted for each stress value. $q_{2}$ can then be varied in order to optimise the general fit (for all $\sigma$ values) of the model to the experimental data. An example of this plot using uniaxial creep data for 316 stainless steel, at $600^{\circ} \mathrm{C}$, is shown by Fig.6. The $q_{2}$ value obtained for the 316 stainless steel, at $600^{\circ} \mathrm{C}$, is included in Table 2. This time marching procedure used is a Forward Euler method, which provides $1^{\text {st }}$ order accuracy, however high order methods, such as $4^{\text {th }}$ order Runge-Kutta method could also be used in order to provide further accuracy to the solution. 


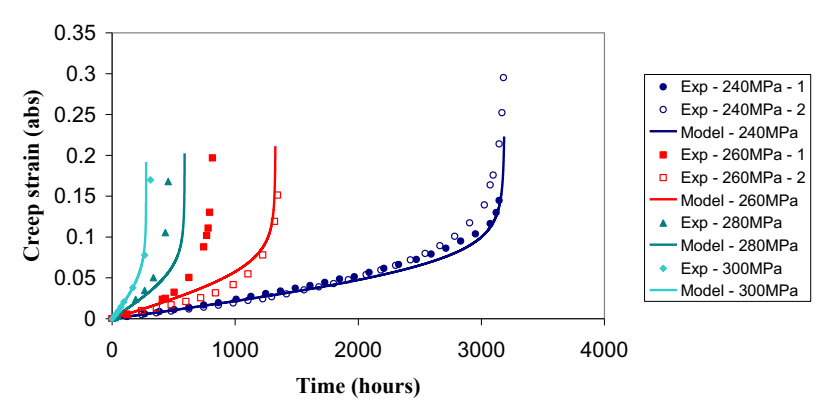

Figure 6. Comparison of the Liu and Murakami creep damage model to uniaxial, experimental creep data.

\subsubsection{Multiaxiality parameter, $\alpha$}

\section{Existing methods used to determine the a-value for a material}

Specimens such as notched bars are tested, under steady load conditions and the failure time obtained. A series of $F E$ analyses with a range of $\alpha$-values, are performed with the same geometry and loading as used in the experimental tests. The material properties for the Liu and Murakami model $\left(C, n_{2}, D, p\right.$ and $\left.q_{2}\right)$, obtained from uniaxial test data, are used for each calculation. The results of these $F E$ calculations are then interpolated to give the $\alpha$-value which results in the same failure time as that of the experimental notched bar test. The average $\alpha$-value obtained for tests with a range of load levels is then taken as the 'best-fit' $\alpha$-value. This process is capable of giving $\alpha$-values which can be used with confidence when the triaxial stress-state in the component under investigation is similar to that in the notch region of the specimens used to determine the $\alpha$-values. However, crack tips have particularly severe multiaxial stress-states and stress magnitudes and hence the damage regions tend to grow in a 'crack-like' manner. Hence, for these situations, it would be advantageous for the $\alpha$-value to be obtained from tests of specimens containing cracks. This novel approach has been adopted in this work.

\section{A novel approach for determining a-values for use} in crack growth predictions

Existing data for 316 stainless steel [7] was chosen to assess the novel approach for determining $\alpha$-values particularly relevant to crack tip type situations. Three $C T$ creep crack growth experiments were carried out [7] with constant loads of $6.977 \mathrm{kN}$ (specimen 106), $7.476 \mathrm{kN}$ (specimen 107) and $8.522 \mathrm{kN}$ (specimen 92), as shown in Table 1. Fig.7 shows photographs of the fracture surfaces of the tested $C T$ specimens, where these specimens are being viewed in the $z-x$ plane as shown in Fig.8. It can be seen that the creep cracks have grown further in the center of the specimen than at the edges, this phenomenon is known as "tunneling". Specimen 92 (Fig.7) has been annotated in order to show the various stages of crack growth for each test. Region 1 shows the starter notch, region 2 shows the initial (fatigue) crack, region 3 shows the creep crack, which gives the valuable part of the test data in the context of this work, region 4 shows further fatigue cracking performed post-test in order to fracture the specimen for analysis and region 5 shows the end region where the specimen was torn open and fractured. It is the data obtained during region 3 which is used to obtain the multiaxial stress-state parameter, $\alpha$, for use in $F E$ analyses of small thumbnail cracks in large cross-sectional rectangular bars.

Table 1. Summary of the CT experimental test details $(T=$ $600^{\circ} \mathrm{C}=$ constant) $[7]$.

\begin{tabular}{cccc}
\hline Test no. & Specimen no. & $\begin{array}{c}\text { Load } \\
(\mathrm{kN})\end{array}$ & $\begin{array}{c}\text { Test duration } \\
\text { (hours) }\end{array}$ \\
\hline 1 & 92 & 8.522 & 168 \\
2 & 106 & 6.977 & 892 \\
3 & 107 & 7.476 & 504 \\
\hline
\end{tabular}



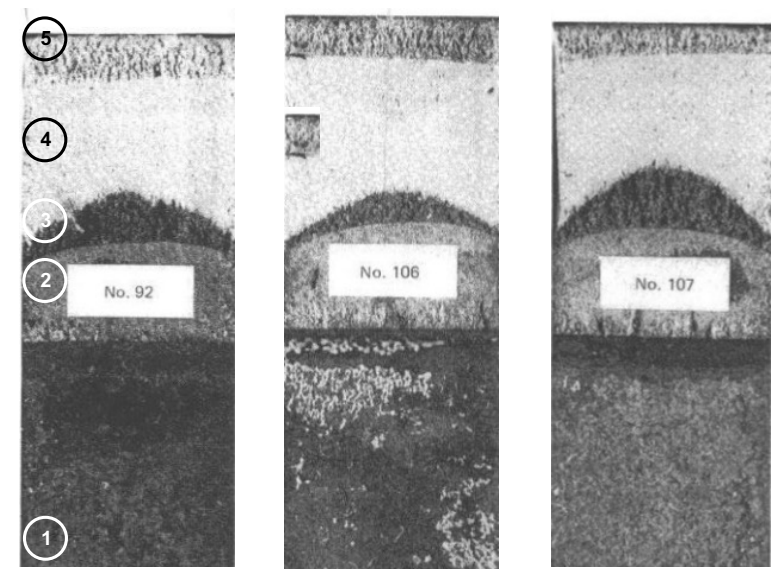

Figure 7. Photographs of creep cracked CT specimens, tested at $600^{\circ} \mathrm{C},[7]$ viewed in the z-x plane (see FIG.8).

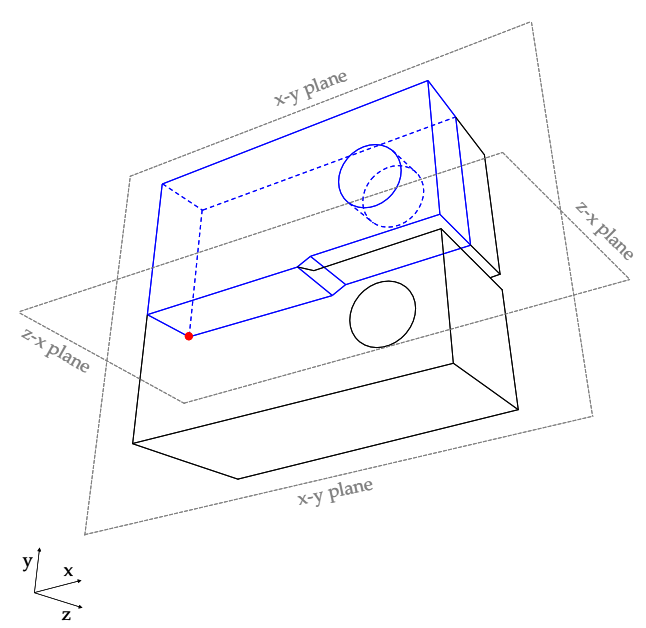

Figure 8. Diagrammatic representation of a CT specimen with the planes of symmetry indicated.

To illustrate the novel method proposed in this paper for determining the $\alpha$-value appropriate for creep crack growth conditions, $C T$ test number 3 (Table 1) with a load of $7.476 \mathrm{kN}$ will be used (i.e. specimen 107). A series of $F E$ calculations with different $\alpha$-values (using all of the other required model constants, already obtained from uniaxial data) were carried out until the average crack length, $a$, obtained in the experiment was achieved, see Fig.7. The time to achieve the crack length, $t_{a}$, is plotted against the $\alpha$-value used, as shown in Fig.9, and a curve is fitted to the data. The experimental $t_{a}$ value of 504 hours is used to interpolate the data in Fig.9, in order to obtain the required $\alpha$-value. It should be noted that the element type used in all analyses presented within this chapter is the 8-noded linear 'brick' type element. All of the $F E$ analyses were carried out by using ABAQUS [10].

Repeating the above process for each load level (i.e. each test/specimen) produces a set of $\alpha$-values. The average of these $\alpha$-values is taken to be the 'best-fit' $\alpha$-value.

Determining the $\alpha$-value in this way (i.e. from severely multiaxial states) means that the resulting value is directly applicable to equally severely multiaxial conditions, such as other crack tip geometries. Previous methods which have used data from specifically biaxial (or other more simplified multiaxial) stress-states to determine the multiaxiality material constant may not produce an $\alpha$-value which is able to deal with such severe stress-states with the same accuracy.

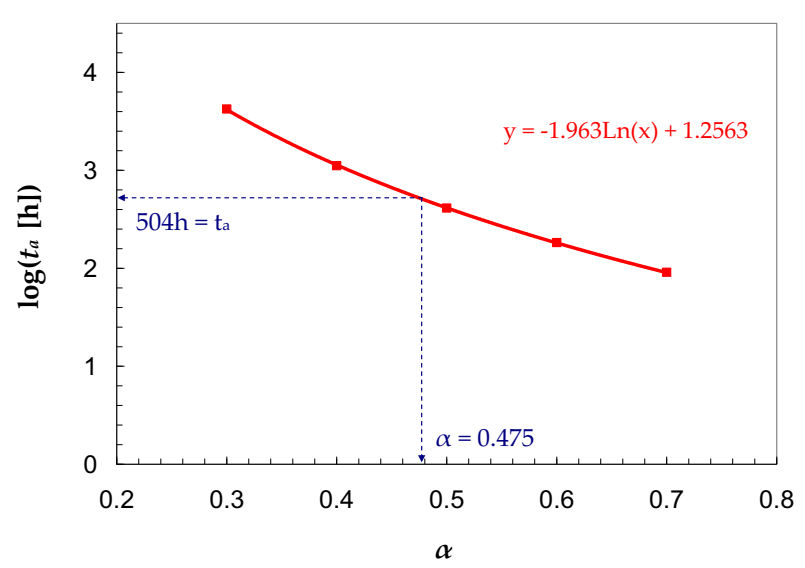

Figure 9. Typical $\alpha$ determination from CT test data, using a logarithmic fitting.

A typical 3-dimensional $F E$ mesh and 0.99 damage zone for the $C T$ specimen geometry used is shown in Fig.10. It should be noted that although an upper damage value of 1 is used in the derivation of 
the model (), a value of 0.99 has been used within all modelling to show complete damage (or crack growth) within the material. This is due to the significant affect this has on maintaining a manageable time step size during the analysis and is justified by the insignificant effect on the accuracy of the analysis results. Due to there being two axes of symmetry in a $C T$ specimen, only one quarter of the specimen has been modelled, with the appropriate boundary conditions applied. These axes of symmetry are shown in Fig.8, where the quarter of the specimen which has been modelled is highlighted in blue. The applied boundary conditions must have the same effect as if the rest of the specimen were present. These boundary conditions on the $C T$ quarter specimen therefore, are complete displacement constraint of the face intersected by the $x-y$ plane in the $z$-direction (see Fig.8), complete displacement constraint of the remaining (i.e. undamaged, as this is the plane were the crack is growing) face intersected by the $z-x$ plane in the $y$-direction and complete displacement constraint of the point shown by the red dot in Fig. 8 in the $x$-direction (this final constraint is actually exerted on the specimen by the test machine, not the specimen itself).

The $\alpha$-value which gave the best overall fit to all of the experimental creep crack growth test data for 316 stainless steel at $600^{\circ} \mathrm{C}$ was found to be 0.478 . The $\alpha$-value and other constants form a complete constant set used for the subsequent $F E$ results $(C T$ and thumbnail) presented. It can be seen from Fig.10 (as well as Fig.14), that the prediction also includes the 'tunnelling' effect observed in the experiments as well as accurate average crack lengths. It is also worth noting that, in reality, compressive stresses would be present at the position indicated by the red dot in Fig.8. Due to this causing problems within the analyses, a condition has been used such that if $\sigma_{r}<0$ then $d \omega=0$ (i.e. only positive rupture stresses are responsible for damage accumulation).

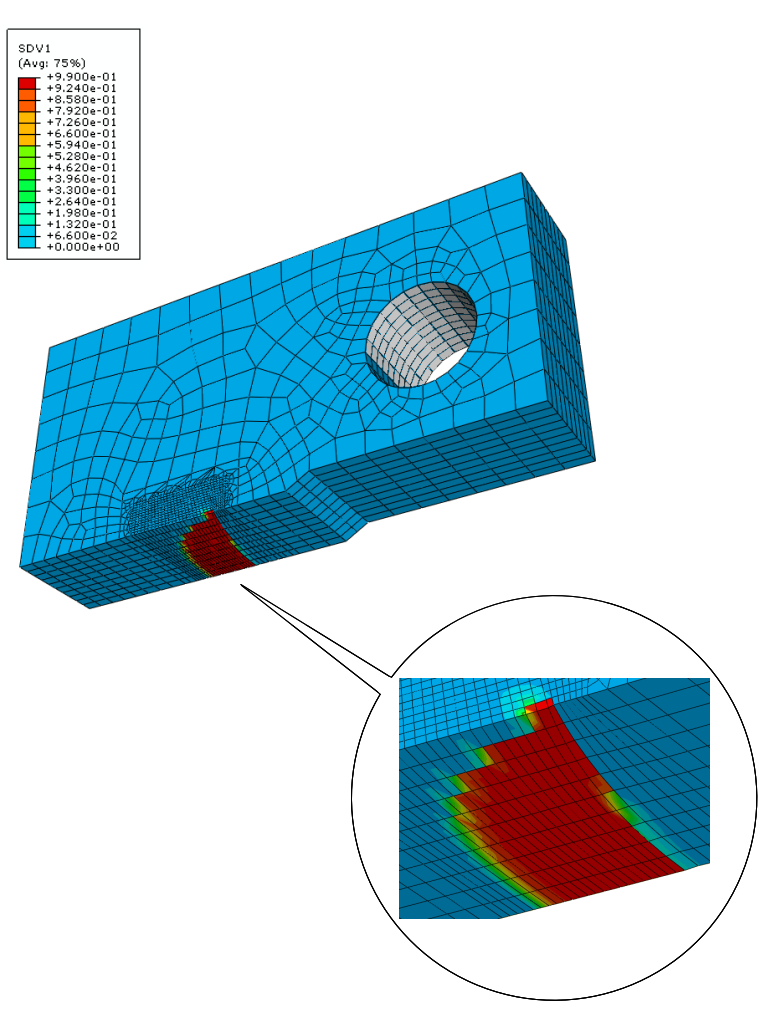

Figure 10. 3D CT specimen FE mesh and damage contour.

Table 2. Complete Liu and Murakami creep-damage model material constant set for 316 stainless steel at $600^{\circ} \mathrm{C}$ (stress in MPa and time in hours).

\begin{tabular}{cccccc}
\hline$C$ & $n_{2}$ & $D$ & $p$ & $q_{2}$ & $\alpha$ \\
\hline $1.472 \mathrm{e}-29$ & 10.147 & $2.73 \mathrm{e}-30$ & 10.949 & 6.35 & 0.478 \\
\hline
\end{tabular}

\section{Results}

Although ABAQUS FE software [10] has the capacity for performing creep analyses using several 'built-in' creep laws, it is not able to perform analyses based on the Liu and Murakami damage model. Therefore, in order to perform $F E$ simulations using models such as the Liu and Murakami damage model, it is necessary to produce a user defined subroutine for the implementation of the model. Such a user defined 
subroutine has been produced using the Fortran computing language for the simulations presented in this paper.

\subsection{Compact tension specimen}

For each of the specimen tests summarised in Table 1, FE calculations were carried out with the material constants defined in Table 2. The analyses were carried out for the test durations used of the corresponding experiments.

Fig.11 shows comparisons of the $F E$ crack growth predictions with the experimental results for the $C T$ specimen geometry shown in Fig.2. These comparisons are made by showing photographs of the symmetric halves of the tested specimens next to the predicted $F E$ damage contours viewed in the $z-x$ plane (see Fig.8) as shown in Fig.11.

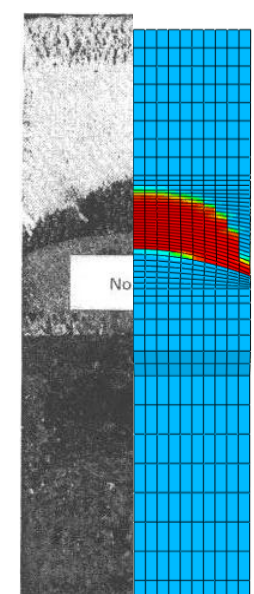

(a)

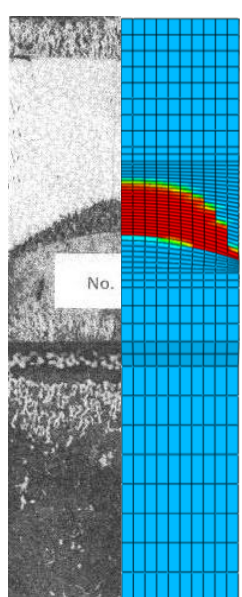

(b)

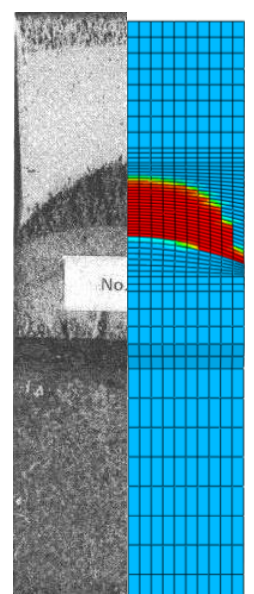

(c)
Figure 11. Tested specimen photo to predicted FE damage contour comparisons viewed in the z-x plane (see FIG.8) (a) Specimen 92, (b) Specimen 106, and (c) Specimen 107.

It can be seen that the overall correlation of the experimental and $F E$ results is good. This is not surprising because, the multiaxial constant, $\alpha$, was determined using the $C T$ specimen crack growth data.
However, the fact that the 'tunnelling' characteristic is well modelled gives further weight to the validity of the method for predicting creep crack growth.

\subsection{Thumbnail crack specimen}

The analyses presented in section 0 , for the $C T$ specimen geometry, were repeated for the thumbnail crack geometry. Five thumbnail creep crack growth tests were carried out under constant loads of $78.7 \mathrm{kN}$ (specimen 112), $90.7 \mathrm{kN}$ (specimen 118), $90.8 \mathrm{kN}$ (specimen 114), 91.7kN (specimen 117) and $102.3 \mathrm{kN}$ (specimen 115) as shown in Table 3. Fig.12 shows photographs of the tested thumbnail crack specimens as viewed in the $z-x$ plane (see Fig.13); specimen 114 has been annotated in order to show the various stages of crack growth for each test. Region 1 shows the starter notch, region 2 shows the initial (fatigue) crack, region 3 shows the creep crack, which again gives the valuable part of the test data in the context of this work, region 4 shows further fatigue cracking performed post-test in order to fracture the specimen into two pieces and region 5 shows the end region where the specimen was torn open and fractured.

Table 3. Summary of the thumbnail crack geometry experimental test details $\left(\mathrm{T}=600^{\circ} \mathrm{C}=\right.$ constant $)$ [7].

\begin{tabular}{cccc}
\hline & & & Test duration \\
Test no. & Specimen no. & Load $(\mathrm{kN})$ & (hours) \\
\hline 1 & 112 & 78.7 & 1176 \\
2 & 114 & 90.8 & 2760 \\
3 & 118 & 90.7 & 1200 \\
4 & 117 & 91.7 & 504 \\
5 & 115 & 102.3 & 504 \\
\hline
\end{tabular}


(a)

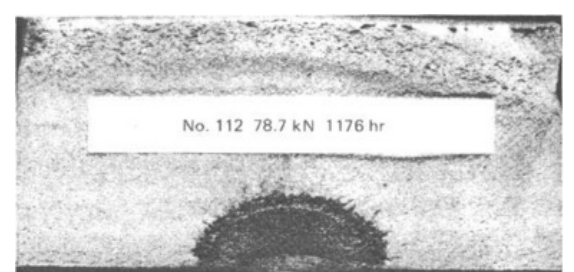

(b)

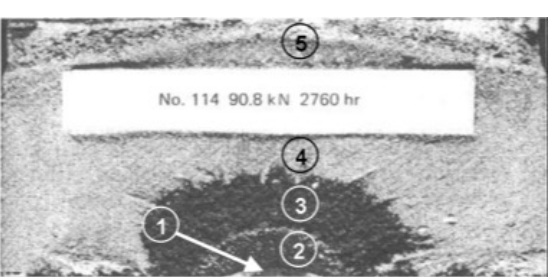

(c)

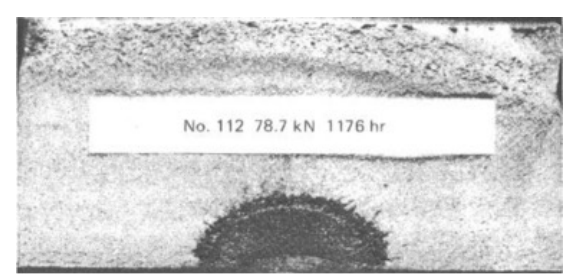

(d)
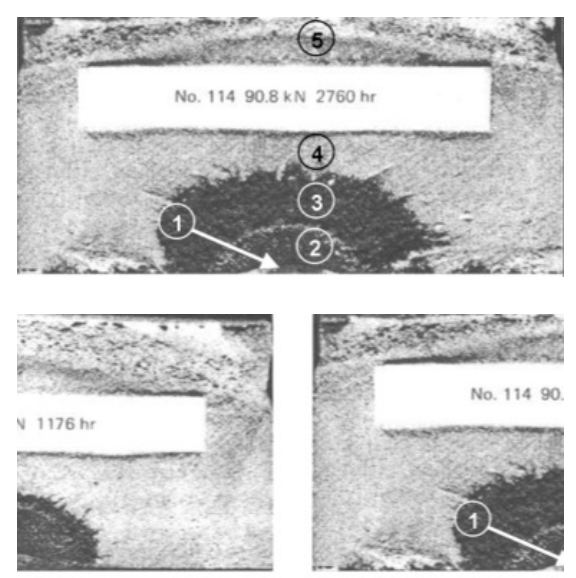

(e)

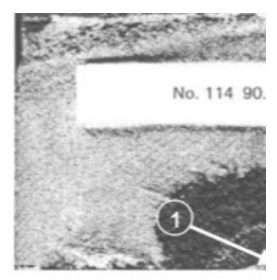

Figure 12. Photographs of creep cracked 316 stainless steel thumbnail crack specimens [7] viewed in the z-x plane (see FIG.13).

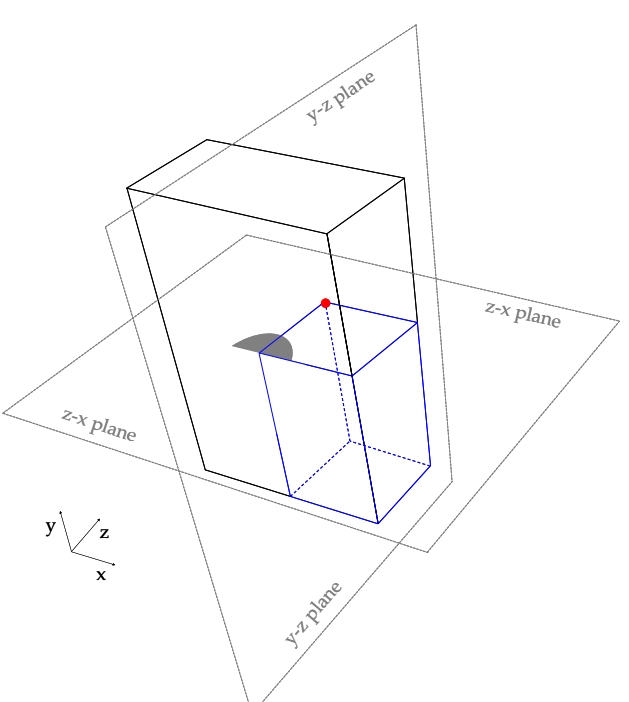

Figure 13. Diagrammatic representation of the

gauge-section of a thumbnail crack specimen with the planes of symmetry indicated.

Fig. 14 shows an example of the $3 D$ mesh used for the thumbnail crack growth simulations and the resulting 0.99 damage zone. As with the $C T$ specimens, due to there being two axes of symmetry in a thumbnail crack specimen, only one quarter of the specimen has been modelled, with the appropriate boundary conditions being applied. These axes of symmetry are shown in Fig.13 in which the quarter of the specimen which has been modelled is highlighted in blue. Again, the applied boundary conditions must have the same effect as if the rest of the specimen were present. These boundary conditions on the modelled quarter of the thumbnail specimen therefore, are complete displacement constraint of the face intersected by the $y-z$ plane in the $x$-direction (see Fig.13), complete displacement constraint of the remaining (i.e. undamaged, as this is the plane were the crack is growing) face intersected by the $z-x$ plane in the $y$-direction and complete displacement constraint of the point shown by the red dot in Fig.13 in the $z$-direction (this final constraint is actually exerted on the specimen by the test machine, not the specimen itself). 


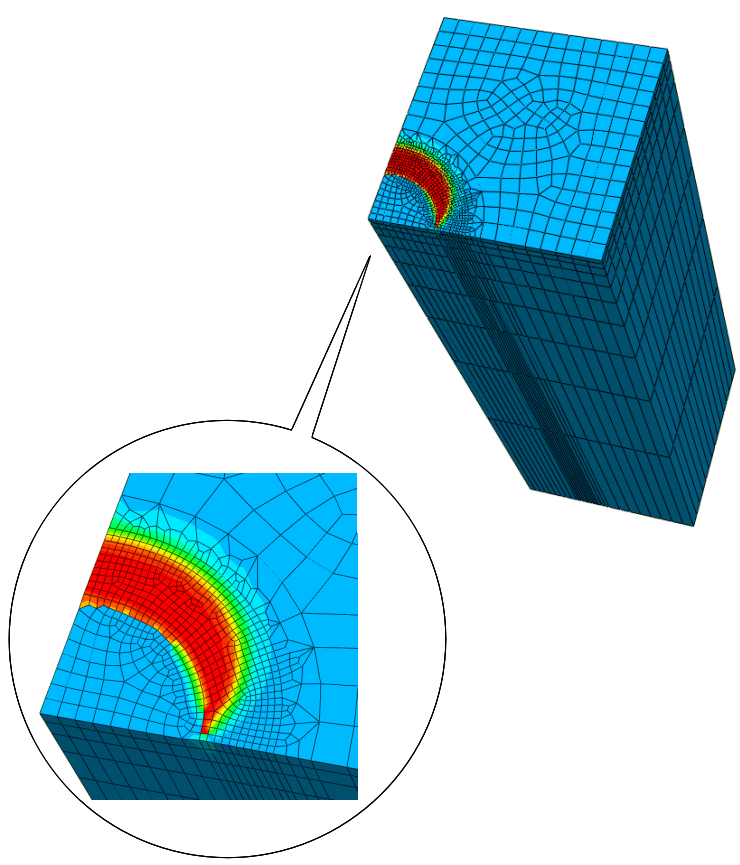

Figure 14. 3D thumbnail crack specimen FE mesh and damage contour.

For each of the specimen tests summarised in Table 3, FE calculations were carried out with the material constants defined in Table 2. Comparisons of the experimental results with the $F E$ creep crack growth predictions for the five thumbnail specimens are shown in Fig. 15 from which it can be seen that the correlation is excellent. These comparisons are made by showing photographs of the symmetric halves of the tested specimens next to the predicted $F E$ damage contours viewed in the $z$ - $x$ plane (see Fig.13).
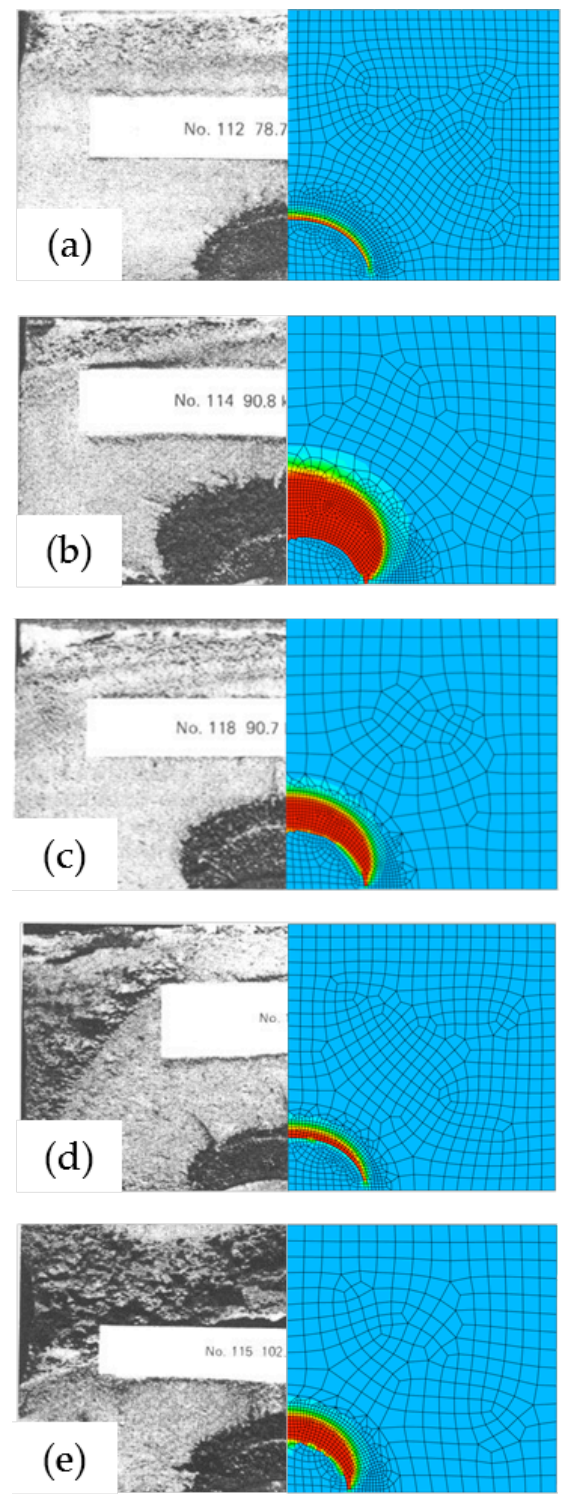

Figure 15. Tested specimen photo to predicted FE damage contour comparisons viewed in the $z$-x plane (see FIG.13)

(a) Specimen 112, (b) Specimen 114, (c) Specimen 118, (d) Specimen 117, and (e) Specimen 115.

Damage simulation within $F E$ analyses is highly mesh-sensitive. Fig.16 shows how the crack length at the axis between the experimental photograph and the $F E$ contour in Fig. 15 varies with element size. 


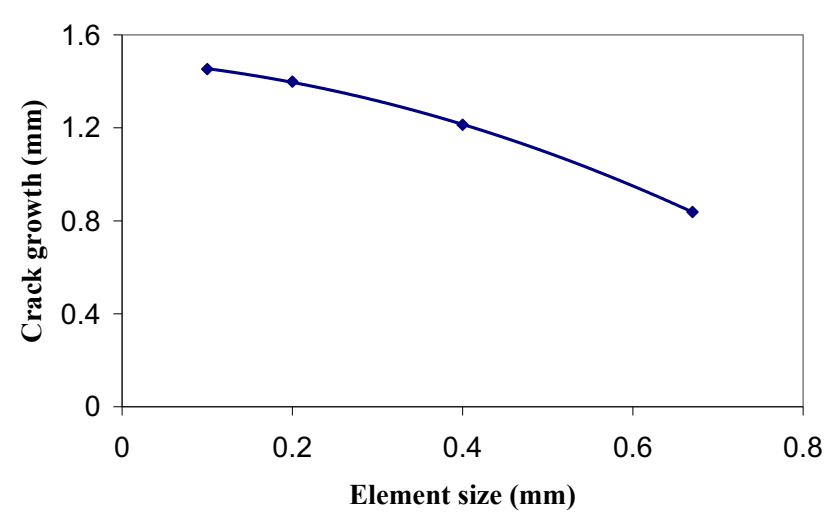

Figure 16. Crack growth vs. element size for thumbnail crack growth predictions (showing mesh sensitivity).

It can be seen from Fig.16 that as the mesh becomes finer, the predicted solution converges towards the correct solution. The difference between the predictions from the two finest meshes $(0.1 \mathrm{~mm}$ and $0.2 \mathrm{~mm}$ element size within the vicinity of the crack tip) is $3.7 \%$. Due to this small difference, the solution can be considered to have converged. Also, due to the small \% difference, in order to balance the accuracy of solution and time of calculation, a mesh using an element size of $0.2 \mathrm{~mm}$ was chosen.

\section{Discussion and future work}

The Liu and Murakami model [6] is suitable for use in predicting creep crack growth. Excellent predictions have been achieved for 316 stainless steel at $600^{\circ} \mathrm{C}$, demonstrating the validity of the creep-damage mechanics-based $F E$ approach as a useful tool for creep crack growth modelling.

A comprehensive procedure for the determination of the material constants for the Lui and Murakami creep damage model, based on experimental data has been described and implemented for 316 stainless steel at $600^{\circ} \mathrm{C}$. A novel method for determining the multiaxiality parameter, $\alpha$, has been introduced.

The closely correlated comparisons of experimental data for thumbnail crack specimens with
FE predictions validate the general applicability of the method.

Further work includes similar experimentation and material modelling but for materials specifically used in high temperature regions of aeroengines and for other types of cracked specimen geometries such as specimens with corner cracks.

\section{Acknowledgments}

The authors would like to thank the EPSRC and the University of Nottingham for the funding through a Doctoral Training Programme and a PhD Plus scheme. Thanks are also given to Dennis Cooper for his skilful technical support within the experimentation.

\section{References}

[1] R. K. Penny and D. L. Marriott, "Design for Creep", McGraw-Hill, Liverpool, 1971.

[2] T. H. Hyde, W. Sun A. A. Becker, "Creep crack growth in welds: A damage mechanics approach to predicting initiation and growth of circumferential cracks in $\mathrm{CrMoV}$ weldments", Int. J. Pres. Ves. \& Piping 78 (2001), 765-771.

[3] B. Dogan and B. Ptrovski, "Creep crack growth of high temperature weldment." International Journal of Pressure Vessel and Piping 78 (2001), 795-805.

[4] L. M. Kachanov, "Theory of creep (english translation edited by A. J. Kennedy)", National lending library, Boston Spa. (1960).

[5] Y. N. Robotnov, "Creep Problems of Structural Members (english translation edited by F. A. Leckie)", North Holland, Amsterdam (1969).

[6] Y. Liu and S. Murakami, "Damage Localization of Conventional Creep Damage 
Models and Proposition of a New Model for

Creep Damage Analysis", JSME International Journal 41 (1998), 57-65.

[7] T. H. Hyde, "Creep crack growth in 316 stainless steel at $600^{\circ} \mathrm{C} "$, High Temperature Technology 6 (1988), no. 2, 51-61.

[8] D. R. Hayhurst, P. R. Dimmer and C. J. Morrison, "Development of continuum damage in the creep rupture of notched bars", Phil. Trans. R. Soc. Lond. (A) 311 (1984), 103-129.
[9] G. A. Webster, S. R. Holdsworth, M. S. Loveday, I. J. Perrin, K. Nikbin, H. Purper, R. P. Skelton and M. W. Spindler, "A code of practice for conducting notched bar creep rupture tests and for interpreting the data", $J$. Fatigue and Fatigue of Eng. Materials and Struct. 24 (2004), 319-342.

[10] Simulia, "Abaqus CAE User's Manual", ver. 6.7, 2007. 